\title{
Pharmacotherapy in dual diagnosis
}

\author{
Ilana B. Crome \& Tracey Myton
}

Abstract The prevalence of coexisting substance misuse and psychiatric disorder (dual diagnosis, comorbidity) has increased over the past decade, and the indications are that it will continue to rise. There have simultaneously been unprecedented developments in the pharmacological treatment of alcohol, opiate and nicotine misuse. Here we evaluate the evidence on the use of some of these treatments in dual diagnosis (with psychotic, mood and anxiety disorders). The evidence base is limited by the exclusion of mental illness when pharmacological agents for substance misuse are evaluated and vice versa. We set the available information within the context of the psychosocial management of comorbid substance misuse and mental illness, and the framework for service delivery recommended by UK national policy.

In the previous issue of APT Mohammed Abou-Saleh (2004) discussed the management of patients with dual diagnosis, with particular reference to current government policy in the UK.

In 1980, Robin Murray raised the question 'Why are the drug companies so disinterested in alcoholism?' (Murray, 1980). Since then, we have witnessed the evolution of a 'specialist addiction field' (Edwards, 2002), including rapid developments in pharmacological treatments for problem use of alcohol, opiates and nicotine. In this review we discuss some of these pharmacological agents and then summarise the evidence on the treatment of combined or coexisting disorders, also described as 'dual diagnosis' (e.g. Banerjee et al, 2002; Crawford et al, 2003).

\section{Dual diagnosis: a definition}

'Dual diagnosis' is one of a number of terms and phrases (Box 1) used to refer to people who have coexisting problems of mental disorder and substance misuse (including alcohol, nicotine and illicit drugs). It is also applied to people with two coexisting conditions, for example learning disability and mental disorder (Banerjee et al, 2002), although it does not take that meaning in this article.

The group of people with dual diagnosis is heterogeneous, with complex, changing needs. They may have had previous traumatic experiences such as childhood sexual abuse, bullying at school or a broken and dysfunctional family life. Furthermore, mental disorder and substance misuse sit on separate dimensions, each with its own continuum of severity. 'Dual diagnosis' covers someone with bipolar disorder who is also alcohol dependent, and someone who has schizophrenia and smokes cannabis a few times a week. As a result of this complexity, numerous operational definitions may be applied in different clinical and social settings, thus complicating and confusing communication.

A patient labelled with a dual diagnosis can face prejudice and stigma from health care and other professionals, who might question the capacity of dually diagnosed individuals to respond to care.

These difficulties raise the question of which definitions and terminology to use. While acknowledging the problems inherent in the term and concept of dual diagnosis, we have chosen to use it as shorthand to describe patients with coexisting problems of mental disorder and substance misuse (of drugs, alcohol or nicotine).

\section{Box 1 Alternatives to the term dual diagnosis}

- Co-occurring problems of substance misuse and mental disorder

- Comorbidity of substance misuse and mental disorder

- Mental illness and chemical abuse (MICA)

- Chemical addiction and mental illness (CAMI)

- Co-occurring addictive and mental disorders (COAMD)

Ilana Crome is Professor of Addiction Psychiatry and Academic Director of Psychiatry, Keele University Medical School (Academic Psychiatry Unit, Keele University Medical School (Harplands Campus), Hilton Road, Harpfields, Stoke on Trent ST4 6TH, UK. E-mail: pca03@keele.ac.uk), and a consultant in addiction for North Staffordshire Combined Healthcare NHS Trust. Since the 1980s she has been directly and actively involved in national clinical research, training and policy developments in the dual diagnosis field both nationally and internationally. At the time of writing this article Tracey Myton was a specialist registrar in addiction psychiatry with Alcohol and Drugs North West, part of Bolton, Salford and Manchester Mental Health Trust. Her special interests are dual diagnosis, young people and treating substance misusers, particularly women, in the prison setting. She intends to become a consultant in addiction psychiatry. 


\section{Prevalence}

The classic Epidemiologic Catchment Area Study in the USA (Regier et al, 1990) demonstrated that $47 \%$ of people with schizophrenia misused substances: alcohol (37\%), cannabis $(23 \%)$ and stimulants or hallucinogens (13\%). Of those with an affective disorder, $32 \%$ had a comorbid substance use disorder. Of those with social anxiety disorder, $22 \%$ had a lifetime prevalence of alcohol dependence or misuse, while among the opiate-dependent population, lifetime rates of anxiety disorders were $6.1 \%$ in men and $10.7 \%$ in women.

In the UK, clinically based population and longitudinal studies consistently demonstrate the prevalence of substance misuse in psychiatric patients (Crawford et al, 2003). In the most recent national comorbidity study, the prevalence of dependence on any drug was $4 \%$, of which cannabis dependence was reported most often (3\%) (Coulthard et al, 2002). The prevalence of drug-taking during the previous year by 16 - to 64 -year-olds had increased from $5 \%$ to $12 \%$ since 1993 . Four per cent of those who had ever used drugs (an estimated $27 \%$ of the total population surveyed) had experienced an accidental overdose.

Men (11\%) were more likely than women (7\%) to report heavy smoking; those aged 20-24 had the highest prevalence, at $44 \%$. Similarly, men $(12 \%)$ were more likely than women $(3 \%)$ to be dependent on alcohol. In general, people who engaged in substance misuse were more likely to be visiting their general practitioner, receiving treatment or accessing mental health services.

It should be noted that lifetime prevalence of substance misuse and mental illness does not necessarily indicate their coexistence at any time. In the only study on comorbidity in primary care in the UK, Frischer et al (2004) addressed this point by counting patients whose diagnoses of substance misuse and psychiatric illness co-occurred within a calendar year. Over the study period (1993-1998) annual prevalence of dual diagnosis in England and Wales increased by $62 \%$ (from 24226 to 39296 ). The rates of comorbid psychosis, schizophrenia and paranoia increased by $147 \%, 128 \%$ and $144 \%$ respectively. By 2003, in a typical general practice 11 potentially chronic comorbid cases were likely to be encountered during the year. This growing epidemic has major implications for general practitioners' workload, as primary care resources in mental health are already thinly spread and are not integrated to deal with comorbidity (Frischer et al, 2004).

Drug problems are associated with smoking and alcohol consumption, constituting dual, or even triple, dependencies (Best et al, 2000). These conditions result in multiple social, physical and psychological complications, which cluster together and with which patients present to accident and emergency, paediatric, geriatric, general medical and surgical, and general psychiatric departments, to primary care and, of course, to specialist addiction units (e.g. Gfroerer et al, 2003).

\section{Government policy}

Comorbidity leads to more frequent recurrence of the mental disorder, greater time spent in hospital, increased violence, homelessness and alienation from families and carers. Once abstinent, however, the dual diagnosis group may have a better prognosis than do non-substance misusers with similar rates of hospitalisation (Granholm et al, 2003).

'Drug problems', especially in young people, remain high on the UK political agenda. The national service frameworks for mental health, children, young people and families, older people, coronary heart disease and cancer are all directly or indirectly related to addiction problems (e.g. Abdulrahim et al, 2001). In addition, there is now a national antismoking strategy (Department of Health, 2003) and an alcohol strategy is being prepared.

Policy guidance on the implementation of dual diagnosis services in England and Wales, and in Scotland, is available (Department of Health, 2002; Scottish Executive, 2003). The Dual Diagnosis Good Practice Guide (Department of Health, 2002) places responsibility for treatment of dual diagnosis clients on mental health services, especially in cases where people have severe and enduring mental health problems. The Scottish Executive's document proposes a five-stepped graded approach to service provision, which would range from a community response, through generic and specialised services, to a highly specialised dual diagnosis service. The focus of this integrated policy is primary care, and it puts more responsibility on primary health care teams to diagnose and treat less complex substance problems.

\section{Treatment works and is cost-effective}

Over the past decade, confidence in the assertion that 'treatment works' has grown immeasurably. To date, about 300 randomised controlled trials (RCTs) have investigated alcohol misuse, 100 tobacco misuse and 60 opiate misuse. It should be noted that some of these studies do not include patients with mental illness: indeed, this diagnosis was often an exclusion criterion, an obvious limitation in evaluating their results. 
Brief psychological therapies carried out by generalists are likely to reap benefits as first-line interventions. However, the more costly intensive psychological interventions (often combined with pharmacological treatment) are effective for those with more complex needs.

Specialist smoking cessation, which costs about $£ 800$ for an intensive 6-week course, is the most costeffective intervention in medicine: smoking-related illnesses cost $£ 16000$ per patient per year (National Institute for Clinical Excellence, 2002). Successful treatment of drug misuse saves the health service $£ 3$ for every $£ 1$ spent. Comorbidity further increases the cost.

\section{The psychosocial context of treatment}

Pharmacological treatment is carried out in the context of attracting, engaging and retaining patients, enhancing their motivation to seek further support, providing the range of specific psychological treatments and medications, and relapse prevention.

Management of any patient should be part of a comprehensive plan following detailed assessment. It should also be noted that, although it is the responsibility of all doctors to provide care for both general health needs and substance-related problems to a reasonable standard, practitioners should be aware of their limitations and seek specialist support if necessary. This is especially the case when treating young people.

Depending on the age of the patient, parental responsibilities, consent to treatment and child protection issues may have to be considered. For young people under the age of 16, explicit consent from a parent or guardian is required.

Psychosocial interventions are relevant throughout because, once detoxification, reduction or maintenance is established, sustained improvement depends on behavioural change. Although most practitioners are, understandably, very concerned about prescribing aspects because of safety issues, in fact the majority of treatment interventions are psychological.

Most general psychiatrists and their teams are in a position to give patients 5-10 minutes of brief advice or information regarding a substance misuse problem. This might include information about the personal risks of substances or about 'safe levels' of drinking, or the provision of self-help materials on ways to stop smoking or on safer injecting practices. The practitioner might also offer the opportunity for the patient to express anxieties, or to discuss the results of screening or blood tests.
Box 2 Coping strategies for patients dealing with substance misuse

- Self-monitoring (keeping a diary about how much they are using)

- Setting limits for use

- Controlling consumption rates (e.g. the number of drinks an hour)

- Learning refusal skills, assertiveness and relaxation

- Making use of new or alternative rewards

- Identifying and challenging negative automatic thoughts that predispose to substance misuse

Although it is often difficult to elicit from the patient an initial indication of 'motivation to change', if this can be established it can contribute substantially towards forming a judgement on how to proceed with regard to pharmacological treatments. Patients need to be encouraged to decide whether abstinence or reduction is their goal.

If abstinence is the goal, and no pharmacological treatment is required, a date on which the patient will stop using substances should be set. Patients should be advised to get rid of alcohol, cigarettes or drugs, and to work on alternative strategies for coping. A number of coping strategies for which there is a substantial and growing evidence base of efficacy are listed in Box 2.

Whether harm reduction, harm minimisation or abstinence is the priority, information about, for example, immunisation, vaccination and contraception, must also form part of the treatment package.

The degree of substance misuse and dependence, as well as associated social, medical and psychiatric problems, may be such that specialist addiction input is required. The practitioner should help the patient to arrange this, and should continue sustained and integrated support and encouragement in collaboration with different agencies.

It should be emphasised to patients and families that 'treatment works', especially if they have had previous negative experiences. This may be pertinent at particular times, for example during pregnancy, or in relation to particular behaviours and lifestyle issues such as prostitution and sex-working. It may be necessary and appropriate to arrange, and even accompany patients to, the first (and maybe more) assessments and consultations with the specialist service(s).

Specialist treatment interventions encompass psychological and pharmacological options. In certain circumstances, patients may have to be admitted to a specialist in-patient unit. This is 
Box 3 Pharmacological interventions for the treatment of substance misuse

Pharmacological interventions may be used:

- in emergencies

- to alleviate withdrawal symptoms so as to achieve abstinence, i.e. detoxification

- to substitute for the drug misused and thus reduce drug-related harm, i.e. stabilisation, reduction, maintenance

- to prevent relapse by reducing alcohol craving

- to treat comorbid psychiatric conditions

- to treat physical consequences

- to treat coexisting physical problems

usually in the case of severe dependence on one or more substances, severe physical illness, serious psychiatric illness, misuse of multiple substances, frequently relapsing substance misuse or unstable social circumstances.

Thus, if more intensive psychological support for the individual or family is required, this will be organised by a specialist and/or general service, on an out-patient basis, in the community, or in conjunction with other services (e.g. general practice or general psychiatric services) or during an assessment or admission.

\section{Treating the substance misuse}

Over the past decade, the capacity to intervene pharmacologically in substance misuse has increased greatly (Box 3). Pharmacotherapy is now available for opiate, alcohol and nicotine misuse (Table 1). Although there is an increasing number of protocols and guidelines for comorbidity in adults (Department of Health, 2002; Scottish Executive, 2003; Slattery et al, 2003), there are few protocols and little guidance specifically related to dual diagnosis in young people, pregnant women and older people. For these special groups, clinical decisions on the potential benefits of pharmacological treatments need to be grounded in good practice, since there is a paucity of well-controlled trials. If medications are prescribed for younger people, this should certainly be initiated and monitored by specialists.

\section{Alcohol}

The drug of choice for alcohol detoxification is chlordiazepoxide. This is a long-acting benzodiazepine and is administered in a withdrawal or detoxification (reduction) regime. A useful rule of thumb is that the initiating dose be the score on the Severity of Alcohol Dependence Questionnaire (SADQ; Stockwell et al, 1979). If, for example, the SADQ score is 30 , then $30 \mathrm{mg}$ chlordiazepoxide three or four times a day should contain withdrawal symptoms. It is important to give sufficient medication. If the patient is oversedated, one dose can be withdrawn. Some practitioners prefer to prescribe diazepam. Anti-epileptics and antiemetics are rarely required, although the former should be given if there is an additional risk factor. Alcohol withdrawal can be monitored using the Clinical Institute Withdrawal Assessment for Alcohol (CIWA-Ar) scale (Sullivan et al, 1989), and the medication reduced over 5-10 days.

Fluids and food should be taken as soon as possible. If not properly controlled, withdrawal can be fatal. Wernicke-Korsakoff syndrome can be confused with delirium tremens, and therefore adequate vitamin supplementation should also be given intravenously. This should be continued for as long as there is clinical improvement. If the syndrome is suspected or diagnosed, doses of up to $500 \mathrm{mg}$ thiamine three times a day for 3 days should be used if necessary, followed by $250 \mathrm{mg}$ daily for 3-5 days (Cook \& Thomson, 1997). WernickeKorsakoff syndrome is a potentially reversible condition in a young person. If a patient is at risk of

Table 1 Pharmacological treatments for substance misuse

Misused substance

$\begin{array}{llll}\begin{array}{l}\text { Treatment } \\ \text { Detoxification }\end{array} & \begin{array}{l}\text { Alcohol } \\ \text { Chlordiazepoxide } \\ \text { Diazepam }\end{array} & \begin{array}{l}\text { Opiates } \\ \text { Methadone } \\ \text { Buprenorphine } \\ \text { Lofexidine } \\ \text { Clonidine }\end{array} & \begin{array}{l}\text { Nicotine } \\ \text { Nicotine replacement therapy } \\ \text { Bupropion }\end{array} \\ \text { Relapse prevention } & \begin{array}{l}\text { Methadone } \\ \text { Buprenorphine }\end{array} & \begin{array}{l}\text { Nicotine replacement therapy } \\ \text { Bupropion }\end{array} \\ & \begin{array}{l}\text { Acamprosate } \\ \text { Disulfiram } \\ \text { Naltrexone }\end{array} & \text { Naltrexone } & \\ & & & \end{array}$


vitamin deficiency, i.e. has weight loss, poor diet or malnutrition, $250 \mathrm{mg}$ thiamine should be given intravenously once a day for 3-5 days (LingfordHughes et al, 2004).

The effects of thiamine deficiency during alcohol detoxification should be treated proactively, and intramuscular vitamin supplementation should be given where possible, as there may be poor absorption of oral vitamins. Patients at low risk should have thiamine $200 \mathrm{mg}$ four times a day and vitamin B compound $30 \mathrm{mg} /$ day.

Carbamazepine has been shown to be effective in preventing withdrawal-related seizures, and interactions with antipsychotic medication (e.g. a reduced seizure threshold) should be considered.

Acamprosate (calcium acetylhomotaurinate) is a relatively new drug which has been reported as reducing craving (Geerlings et al, 1997). Side-effects include skin rash and gastrointestinal problems.

Disulfiram, which interferes with the breakdown of alcohol, resulting in the accumulation of acetaldehyde, produces unpleasant - even fatal effects (e.g. headache, flushing, nausea, vomiting and circulatory collapse) if alcohol is taken. Its longterm effectiveness in adults is not convincing, but this could be due to inadequate dosage and supervision (Besson et al, 1998).

Naltrexone can only be administered on a named patient basis, as it is not licensed for use for alcohol problems in the UK.

Apart from detoxification with benzodiazepines and vitamin supplementation, there is no evidence to support the routine use of other medications at the moment, although there are potentially interesting developments with combined medications, for example naltrexone and acamprosate (Kiefer et al, 2003).

\section{Nicotine}

Two products are available for the treatment of nicotine addiction: nicotine replacement therapy (as chewing gum, spray, skin patch, sublingual tablets and lozenges) and bupropion.

Nicotine replacement therapy is available in the UK over the counter or on prescription. For those under 18 years old it is available 'on the recommendation of a medical practitioner' (National Institute for Clinical Excellence, 2002). It is not recommended during pregnancy and breastfeeding (National Institute for Clinical Excellence, 2002). It may produce localised side-effects, and minor sleep disturbances are common.

Bupropion is available only on prescription. It is not recommended for young people under 18 years old, and it should be avoided in pregnant or breastfeeding women and in patients with a history of fits, with alcohol and other drug misuse problems or with cerebral trauma. Three per cent of those prescribed develop mild reactions (e.g. rash, urticaria or pruritus) and $0.1 \%$ develop hypersensitivity reactions.

Smokers of 10 or more cigarettes a day should normally be encouraged to use nicotine replacement therapy; combinations of nicotine replacement preparations are more effective than a single type. The agreement of the patient's physician should be obtained before nicotine replacement therapy is given to patients who have associated serious medical conditions.

\section{Illicit drugs \\ Opiates}

A range of medications are available for detoxification, stabilisation and reduction. This includes methadone, buprenorphine, clonidine, lofexidine, naltrexone, as well as combinations (e.g. lofexidine and naltrexone). Naloxone is, of course, prescribed as an antidote to overdose, but since it is short-acting, regular monitoring and supervision of the patient's state of consciousness and withdrawal are necessary. Although heroin can be prescribed by licensed practitioners in the UK, this is still an area of controversy which needs to be resolved by RCTs.

A definite diagnosis of 'dependence' is invariably required before substitute medication is initiated. This should take into account clinical examination, history, with corroborating evidence, and results of investigations. In young people, it is extremely important that specialist assessment be organised at the earliest possible stage.

If substitute drugs are prescribed, the consensus is that they should be available on a 'daily pick-up' basis, at least at the start, supervised by the pharmacist or, failing that, by a reliable carer or parent (in the case of those under 18 years old). At present, pharmacists appear more willing to supervise methadone than buprenorphine therapy.

\section{Stimulants and cannabis}

Since substitute medication is not available for cocaine and cannabis, and not commonly used for amphetamines, the administration of general treatment measures is advised. In stimulant withdrawal, patients may become drowsy and depressed, and even suicidal. Regular monitoring of mental state and physical examination are therefore very important. Urine screening should be frequently and randomly carried out. Cannabis withdrawal may result in insomnia, and hypnotic sedatives should be prescribed cautiously. 
Table 2 Selected studies on the pharmacological treatment of psychosis and substance misuse

$\begin{array}{lll}\text { Study } & \text { Substance } & \text { Medication } \\ \text { Buckley et al, 1994 } & \text { Various } & \text { Clozapine } \\ \text { Conley et al, 1998 } & \text { Various } & \text { Olanzapine } \\ \text { Levin } \text { et al, 1998 } & \text { Cocaine } & \begin{array}{l}\text { Flupentixol } \\ \text { Drake } \text { et al, 2000 }\end{array} \\ & \begin{array}{l}\text { Alcohol } \\ \text { Cannabis }\end{array} & \text { Clozapine } \\ \text { Zimmet et al, 2000 } & \text { Various } & \text { Clozapine } \\ \text { Littrell } \text { et al, 2001 } & \text { Various } & \text { Olanzapine } \\ \text { George } & \text { Nicotine } & \text { Clozapine } \\ \text { \& Vessicchio, 2002 } & & \end{array}$

Special groups

Young people

Apart from buprenorphine and, in some instances, nicotine replacement therapy (if recommended by a physician), no drug in the management of alcohol, nicotine or opiate dependence is licensed for use in those under 18 years old. Thus, as mentioned earlier, specialist assessment is essential.

Pregnant women

In pregnancy, alcohol consumption usually decreases as a matter of course. The first RCT of comprehensive assessment and brief interventions demonstrated reductions in both groups (assessment alone and assessment with intervention), but prior abstinence in addition to a brief intervention had the better outcome (Chang et al, 1999). RCTs for nicotine replacement therapy in pregnancy are required. Recent guidance from the National Institute for Clinical Excellence has stated that nicotine replacement therapy can be prescribed for pregnant or breastfeeding mothers (National Institute for Clinical Excellence, 2002). Methadone treatment as part of a prenatal package for opiate-dependent women has been shown to improve birth outcomes and maternal psychosocial function. A neonatal withdrawal syndrome may develop if methadone is used, whereas buprenorphine has been found to be safe and effective in mother, foetus and neonate (Fischer et al, 2000).

Prisoners

Great care must be taken when prescribing substitute medication for someone who has just been released from prison. It is likely that their drug use has been markedly reduced while in custody and therefore their tolerance may have decreased. In other words, a previously prescribed dose of medication may be too high and even fatal.

\section{Treating the psychiatric disorder}

The vast range of pharmacological treatments for psychiatric disorders has been collated by the World Health Organization (Andrews \& Jenkins, 1999). Here, we consider just a few for specific disorders comorbid with substance misuse. However, given the prevalence of such comorbidity and the wide range of psychiatric conditions involved, few studies have investigated pharmacological treatments for dual diagnosis (Table 2).

\section{Schizophrenia and schizoaffective disorder Treatment with clozapine}

There is good evidence to suggest that clozapine reduces substance misuse in patients with schizophrenia. The response to clozapine of patients with treatment-resistant illness and a current or previous history of substance misuse is comparable with that of patients with treatment-resistant illness who do not misuse substances: both groups show reduced psychopathology and improved psychosocial functioning (Buckley et al, 1994; Zimmet et al, 2000). This response may result from an improved mental state or a direct anti-craving effect produced by the drug. The mesolimbic dopamine system is associated with motivational states and is implicated in the reinforcing actions of most drugs of misuse. Nicotine, alcohol and cocaine increase extracellular dopamine levels. Clozapine's greater affinity for $D_{1}$ and lesser affinity for $\mathrm{D}_{2}$ receptors may modulate the activity of the receptors involved in craving.

Clozapine and nicotine dependence Clozapine is particularly beneficial when the drug of comorbid dependence is nicotine. Eighty per cent of people with schizophrenia are smokers, and this contributes to the considerable increase in standardised mortality rate seen in schizophrenia. Nicotine produces a transient improvement in sensory gating and other neuropsychological deficits associated with schizophrenia (George \& Vessicchio, 2002). Clozapine produces similar improvements, but the effects are sustained.

Clozapine and alcohol dependence In patients with a diagnosis of either schizophrenia or schizoaffective disorder and comorbid alcohol misuse, clozapine has been shown to decrease severity of alcohol use and number of drinking days and increase remission of alcohol-use disorders (Drake et al, 2000). This reduction was strongly correlated with a decrease in symptoms of anergia, but not with reduced positive symptoms of schizophrenia. Another study demonstrated that clozapine 
treatment was associated with a reduction in alcohol use in $83 \%$ of patients with comorbid alcohol misuse and schizophrenia (Zimmet et al, 2000). None of the group increased their alcohol consumption while on clozapine. There was a correlation between decreased alcohol use and a reduction in global clinical symptoms.

Clozapine and cannabis misuse Drake et al (2000) also examined clozapine in comorbid cannabis use. The findings were less consistent than those with alcohol, but a reduction in use was demonstrated.

\section{Treatment with other antipsychotics}

Clozapine treatment has disadvantages in terms of adverse effects and the need for regular full blood count monitoring. There is less evidence for the use of olanzapine in dual diagnosis (Conley et al, 1998), although Littrell et al (2001) demonstrated that olanzapine improved both psychosis and substance misuse. A small pilot study examined the effects of flupentixol treatment in schizophrenia complicated by cocaine misuse (Levin et al, 1998). It reported a reduction in both positive and negative symptoms and a decrease in cocaine use in the majority of participants. Flupentixol may be the typical antipsychotic of choice if treatment with an atypical is not possible.

\section{Mood disorders}

In substance misuse, depressive and anxiety symptoms (as well as many psychological symptoms) are often transient and related to the effects of the substances misused, to withdrawal from them or to psychosocial stress associated with the individual's lifestyle. Detoxification often leads to an improvement in mood, as does maintenance therapy in opiate dependence. It is therefore preferable to delay diagnosis of clinical depression until the patient has had a period of abstinence, when it should also be easier to distinguish clinical depression from feelings of misery and unhappiness. Such a delay should be possible in most cases, although if patients have suicidal ideation a judgement may be needed regarding initiation of antidepressant medication before abstinence has been achieved.

\section{Depression and alcohol dependence}

Thirty per cent of those dependent on alcohol fulfil the criteria for a major depressive disorder, and 80\% experience some depressive symptoms. Comorbidity has been shown to increase the risk of suicide. Schuckit et al (1994) demonstrated that few patients presenting with alcohol and depression have a major depressive disorder. It is generally believed that depressive symptoms should not be treated for 2-4 weeks following detoxification from alcohol. It is important to recognise that depressive symptoms increase the risk of relapse, either as a cue to drinking or by causing patients to disengage with relapseprevention treatment. Studies investigating the benefits of prescribing antidepressants to this population have relatively short follow-up times, given that uncomplicated depression should be treated with medication for at least 6 months to reduce relapse.

Most of the available studies (some of which are listed in Table 3) are not standardised for dependent or harmful drinking, or for primary or secondary depression. There are no studies comparing the efficacy of tricyclics with selective serotonin reuptake inhibitors ( SSRIs), or comparing differing

Table 3 Selected studies on the pharmacological treatment of mood and anxiety disorders comorbid with substance misuse

\begin{tabular}{|c|c|c|c|}
\hline Study & Disorder & Substance misused & Treatment/medication \\
\hline Kranzler et al, 1995 & Depression & Alcohol & SSRI \\
\hline McGrath et al, 1996 & Depression & Alcohol & Imipramine \\
\hline Mason et al, 1996 & Depression & Alcohol & Desipramine (withdrawn in the UK) \\
\hline Cornelius et al, 2000 & $\begin{array}{l}\text { Depression and } \\
\text { alcoholism }\end{array}$ & Marijuana & SSRI (fluoxetine) \\
\hline Petrakis et al, 1994 & Depression & Opiates & SSRI \\
\hline Hamilton et al, 1998 & $\begin{array}{l}\text { Depression, methadone } \\
\text { maintenance }\end{array}$ & Opiates & Nefazodone (withdrawn in the UK) \\
\hline Nunes et al, 1998 & Depression & Opiates & Imipramine \\
\hline Schmitz et al, 2001 & $\begin{array}{l}\text { Major depressive } \\
\text { disorder }\end{array}$ & Cocaine & Fluoxetine \\
\hline Malec et al, 1996 & Anxiety & Alcohol & Buspirone \\
\hline Charney et al, 2000 & Anxiety & Benzodiazepines & Reduction regime \\
\hline Hertzman, 2000 & Bipolar disorder & Alcohol & Valproate \\
\hline Brown et al, 2002 & Bipolar disorder & Cocaine & Quetiapine \\
\hline
\end{tabular}


doses of an individual medication. In a population at high risk of suicide, SSRIs have advantages over tricyclics in terms of safety profile.

\section{Tricyclic antidepressants in alcohol misuse}

Desipramine and imipramine have both been shown to be effective medications for depression comorbid with alcohol misuse. A double-blind, placebo-controlled trial of desipramine was carried out by Mason et al (1996) in alcohol-dependent patients who had been abstinent for at least a week. Both depressed and non-depressed individuals were recruited to see whether desipramine had an effect on relapse rate, irrespective of its antidepressant properties. Desipramine showed significant benefits over placebo in relapse rate $(8.3 \% v .40 \%)$ and also in time to relapse. However, desipramine is no longer licensed in the UK.

A randomised placebo-controlled trial of imipramine followed up patients with depression who were drinking excessively but who did not necessarily have a history of dependence (McGrath et al, 1996). Participants were required to have had depression prior to the alcohol misuse or during at least 6 months of sobriety. Treatment lasted 12 weeks, with maximum doses of $300 \mathrm{mg}$ of imipramine. Depression improved in the imipramine-treated group, but there was no significant decrease in either drinking days or alcohol consumed per drinking day. There was no adverse interaction between imipramine and alcohol, but $13 \%$ of those treated with imipramine withdrew from the trial because of adverse side-effects.

\section{SSRIs in alcohol misuse}

Selective serotonin reuptake inhibitors both decrease depressive symptoms and reduce alcohol consumption in patients comorbid for depression and alcohol misuse.

A 1-year randomised, double-blind, placebocontrolled trial of fluoxetine in patients with comorbid depression and alcohol dependence demonstrated the superiority of fluoxetine (Cornelius et al, 2000). The paper does not state the dose of fluoxetine prescribed, but no significant adverse effects were reported. The number of days of drinking to intoxication was reduced significantly. Total drinking days were also reduced by treatment with fluoxetine, but not statistically significantly so. None of the patients in either group was entirely abstinent. There was no improvement between 3- and 12-month outcomes. These findings suggest that continuing antidepressant medication for 12 months should be recommended in this group.

Kranzler et al (1995), however, report that fluoxetine in doses of up to $60 \mathrm{mg}$ used for relapse prevention in the absence of depression was not effective in reducing drinking.

\section{Bipolar affective disorder and substance misuse}

Bipolar disorder is most commonly comorbid with alcohol misuse. There is some evidence that treatment with sodium valproate is beneficial. Anticonvulsants may reduce membrane instability in the presence of substance misuse. A retrospective study of valproate used to treat bipolar disorder in patients with concomitant substance misuse demonstrated that $50 \%$ reduced their substance misuse (Hertzman, 2000). A study by Brown et al (2002) reported improvement in cocaine users with bipolar disorder: see 'Cocaine and depression' below.

\section{Depression and opiate dependence}

Tricyclic antidepressants Imipramine has been shown to be effective in reducing depressive symptoms in a placebo-controlled study of opiatedependent patients receiving methadone maintenance (Nunes et al, 1998). The participants had depression that either preceded the onset of regular substance misuse or had lasted at least 3 months after the start of misuse. Maximum doses of $300 \mathrm{mg}$ imipramine were used (mean of $268 \mathrm{mg}$ ). It should be noted that methadone increases serum levels of tricyclics. Participants were followed up for 12 weeks. Abstinence was achieved in $14 \%$ of the imipramine group and $2 \%$ of the placebo group. There was an association between improvement in depressive symptoms and reduced substance misuse, but causality could not be inferred. The substance misuse improved before the depression in more than half of responders, suggesting that mood is only one of several factors driving substance use.

SSRIs Petrakis et al (1994) conducted a small pilot study to examine the effects of prescribing fluoxetine to methadone-maintained opiate-dependent patients with either comorbid depression or persistent cocaine use. The mean dose of fluoxetine was $47 \mathrm{mg}$, and the follow-up period was 12 weeks. Frequency of self-reported drug use decreased significantly, and depressive symptoms decreased but not significantly. Participants reported anecdotally that fluoxetine reduced their subjective craving for cocaine.

There have also been case studies describing success with nefazodone in treating depression in methadone-maintained opiate-dependent patients (Hamilton et al, 1998). Nefazodone has recently been withdrawn in the UK.

The prescription of antidepressants should be undertaken very cautiously, and monitored regularly, particularly in light of recent findings on 
deaths due to antidepressants. Deaths involving antidepressants in combination with other drugs are significantly more likely to be those of drug misusers (Cheeta et al, 2004). Prescription should be undertaken within the context of a risk assessment that includes the potential for self-harm, suicidal ideation, intention or behaviour, risk of harm to others, and self-neglect. Predictors of risk behaviour include a previous history of harm to self or others, hopelessness, agitation, command hallucinations, social isolation, recent losses and recurrent psychiatric hospitalisations (Evans \& Sullivan, 2000). A mental health or drug problem that impairs judgement may further heighten risk.

Cocaine and depression Several studies have investigated the treatment of comorbid cocaine addiction and depression (Schmitz et al, 2001; Brown et al, 2002). Fluoxetine and quetiapine were administered, respectively. Fluoxetine failed to produce a response, but the findings were 'promising' for quetiapine in that psychiatric symptoms and cocaine craving improved.

\section{Anxiety disorders}

The relationship between alcohol use and anxiety disorders is not necessarily causal but may be a reflection of neurophysiological factors that give rise to both disorders (Lingford-Hughes et al, 2002). Neurotransmitter and receptor function in the $\gamma$-aminobutyric acid (GABA), serotonin and noradrenaline systems are altered in both. Thus, as with associated depressive symptoms, a period of abstinence following withdrawal or intoxication should elapse, if feasible, before a diagnosis of anxiety is made.

Some of the key studies addressing anxiety disorders comorbid with substance misuse are listed in Table 3.

\section{Treatment of comorbid alcohol dependence and anxiety}

Anxiety is a common symptom of alcohol withdrawal, and many patients presenting for detoxification give a history of agoraphobia or panic disorder. Even with the most thorough history, it is often difficult to distinguish between the disorders. Anxiety disorders cannot be successfully treated while a patient is drinking heavily and so detoxification should be the first stage of treatment, followed by reassessment of the anxiety symptoms. Psychological treatment in the form of anxiety management groups is part of the treatment offered by in-patient detoxification units and community alcohol teams.
In terms of medication, there is some evidence for the effectiveness of buspirone in this group. Malec et al (1996) reviewed five studies, which showed that the main effects of buspirone were an improvement in anxiety symptoms and greater treatment retention. There was no consistent reduction in alcohol consumption, however.

\section{Treatment of comorbid benzodiazepine dependence and anxiety}

Benzodiazepines are used as hypnotics, sedatives or anxiolytics. It is important to recognise that they can produce a withdrawal syndrome - i.e. dependenceeven if low doses have been prescribed (or misused). If taken in combination with other drugs and alcohol, overdose can result (Hawton et al, 1998). Alternatives such as buspirone, zolpidem and zopiclone do not appear to lead to dependence.

There is little evidence concerning the pharmacological treatment of comorbid benzodiazepine dependence and anxiety. Charney et al (2000) examined the treatment of benzodiazepine dependence to evaluate predictors of successful outcome. Although their study did not target a comorbid population, $62 \%$ of the study population had a lifetime diagnosis of an anxiety disorder and 68\% of these had been prescribed benzodiazepines to treat this disorder. It seemed that after chronic use patients no longer experienced any anxiolytic effect. Treatment consisted of out-patient detoxification with reducing doses of diazepam over a maximum of 10 weeks, followed by group and individual work. After 6 months of follow-up they reported a decrease in anxiety symptoms, despite having considerably reduced their benzodiazepine use. Fifty per cent of the participants had maintained abstinence. In terms of predictors of outcome, anxiety did not impede successful treatment.

\section{Monitoring interventions}

It is worth pointing out that some psychiatric patients with comorbid substance misuse achieve stabilisation rapidly. Furthermore, severe mental illness does not necessarily predict worse outcome. However, early unplanned discharge is common in this patient population. Monitoring and compliance, helped by motivational techniques, are vital components of ensuring the value of pharmacological interventions (Hunt et al, 2002).

Socio-economic and emotional aspects are the main challenges to recovery, and case management in the context of integrated community and residential services with assertive outreach has been shown to increase medication compliance with increasing efficiency over time. A newer approach 
is 'sensitive anticipatory action' (Tyrer \& Weaver, 2004). This focuses on medication review as one component of relapse prevention, and on social care planning, which encompasses help with accommodation as well as forward planning to avoid crises.

The appropriateness of a model of service delivery depends on local arrangements. For this reason, the health, social care, housing, criminal justice and voluntary sector agencies of each local area need to reach an operational definition of the patient group and to identify service roles and capacity.

\section{Outstanding critical issues}

There are few controlled studies for such a common and complex area as dual diagnosis. In those that have been published, the robustness of diagnosis varies and some report a reduction in psychiatric symptoms although there was no ICD or DSM diagnosis of psychiatric disorder. Furthermore, in general, primary outcome measures are usually related to the psychiatric disorder and secondary outcomes to the substance of misuse. Some studies do look at the effect of a particular medication on, for example, both mood and drug misuse, and attempt to relate these - rather than make drug use secondary. There is also the need to identify whether the primary objective of the pharmacotherapy is to treat the mental health issue or to alleviate the substance misuse problem (e.g. SSRIs to counter the serotonergic dysfunction of alcohol dependence). Furthermore, some studies evaluated efficacy once patients were drug-free, whereas others were concerned about safety issues, e.g. side-effects of antipsychotics.

Sample selection, small sample sizes, lack of control groups, lack of masking, different outcome measures, reliability of self-report, length of followup, and concomitant psychosocial or pharmacological treatments further complicate the extent to which findings are practically useful. In summary, since the number of standardised studies is limited, it is difficult to draw conclusions and, especially, to make recommendations.

\section{Conclusions}

This is undoubtedly a complicated area, where adequate robust research evidence is just beginning to emerge. An array of pharmacological interventions is available for the treatment of substance use disorders individually. However, these agents have usually been evaluated in patients without comorbid psychiatric disorders. Similarly, although there is a growing number of antipsychotics, antidepressants and anti-anxiety agents, surprisingly few have been tested on comorbid conditions.

It would appear, on the basis of the limited evidence, that clozapine and possibly olanzapine are useful for psychotic disorders complicated by substance misuse. Although imipramine, fluoxetine, doxepin and venlafaxine have been reported to be effective in depressive disorders complicated by different substances of misuse or dependence, it must be stressed that many of the findings of studies are inconsistent. Similarly, results on the effects of buspirone for anxiety and alcohol disorders are mixed.

Decisions need to be made on the basis of a thorough assessment (including risk assessment) of the nature and extent of the mental illness and the substance misuse, the psychosocial environment in which the patient is functioning, as well as of the local multi-specialist and multi-agency arrangements in place for the delivery of care, which includes pharmacotherapies. Patients with a dual diagnosis form a heterogeneous group of often complex individuals with negative experiences of the treatment system, and they are consequently at high risk of significant morbidity and mortality. Practitioners need to be especially vigilant with regard to safety. Keeping a close clinical eye on medication is critical, because the research evidence on risk benefit and cost-effectiveness is not yet forthcoming.

\section{References}

*Abdulrahim, D., Annan, J., Cyster, R., et al (2001) Developing an Integrated Model of Care for Drug Treatment: Promoting Quality, Efficiency and Effectiveness in Drug Misuse Treatment Services. London: National Treatment Agency.

Abou-Saleh, M. T. (2004) Dual diagnosis: management within a psychosocial context. Advances in Psychiatric Treatment, 10, 352-360.

Andrews, G. \& Jenkins, R. (eds) (1999) Management of Mental Disorders (vols. 1 \& 2). London: World Health Organization Collaborating Centres in Mental Health.

*Banerjee, S., Clancy, C. \& Crome, I. B. (eds) (2002) Coexisting Problems of Mental Disorder and Substance Misuse (Dual Diagnosis): An Information Manual (2nd edn). London: College Research Unit and Royal College of Psychiatrists.

Besson, J., Aeby, F., Kasas, A., et al (1998) Combined efficacy of acamprosate and disulfiram in the treatment of alcoholism: a controlled study. Alcoholism: Clinical and Experimental Research, 22, 573-579.

Best, D., Rawaf, S., Rowley, J., et al (2000) Drinking and smoking as concurrent predictors of illicit drug use and positive drug attitudes in adolescents. Drug and Alcohol Dependence, 60, 319-321

Brown, E. S., Nejtek, V. A., Perantie, D. C., et al (2002) Quetiapine in bipolar disorder and cocaine dependence. Bipolar Disorders, 4, 406-411.

Buckley, P., Thompson, P. A., Way, L., et al (1994) Substance abuse and clozapine treatment. Journal of Clinical Psychiatry, 55 (suppl. B), $114-116$.

Chang, G., Wilkins-Hang, L., Berman, S. A., et al (1999) Brief interventions for alcohol use in pregnancy. A randomised trial. Addiction, 94, 1499-1508. 
Charney, D. A., Paraherakis, A. M. \& Gill, K. J. (2000) The treatment of sedative-hypnotic dependence: evaluating clinical predictors of outcome. Journal of Clinical Psychiatry, 61, 190-195.

Cheeta, S., Schifano, F., Oyefeso, A., et al (2004) Antidepressant-related deaths and antidepressant prescriptions in England and Wales, 1998-2000. British Journal of Psychiatry, 184, 41-47.

Conley, R. R., Kelly, D. L. \& Gale, E. A. (1998) Olanzapine in treatment refractory schizophrenic patients with a history of substance misuse. Schizophrenia Research, 33, 95-101.

Cook, C. C. H. \& Thomson, A. D. (1997) B-complex vitamins in the prophylaxis and treatment of Wernicke-Korsakoff syndrome. British Journal of Hospital Medicine, 57, 461465 .

Cornelius, J. R., Salloum, I. M., Haskett, R. F., et al (2000) Fluoxetine versus placebo in depressed alcoholics: a oneyear follow-up study. Addictive Behaviour, 25, 307-310.

Coulthard, M., Farrell, M., Singleton, N., et al (2002) Tobacco, Alcohol and Drug Use and Mental Health. London: Stationery Office.

*Crawford, V., Clancy, C. \& Crome, I. B. (2003) Co-existing problems of mental health and substance misuse (dua diagnosis): a literature review. Drugs: Education, Prevention and Policy, 10 (suppl.), S1-S74.

Department of Health (2002) Mental Health Policy Implementation Guide: Dual Diagnosis Good Practice Guide. London: Department of Health.

Department of Health (2003) National Alcohol Harm Reduction Strategy: Interim Analytical Report. London: Department of Health. http://www.number10.gov.uk/output/ page4498.asp

Drake, R. E., Xie, H., McHugo, G. J., et al (2000) The effects of clozapine on alcohol and drug use disorders among patients with schizophrenia. Schizophrenia Bulletin, 26, 441-449

Edwards, G. (ed.) (2002) Addiction: Evolution of a Specialist Field. Oxford: Blackwell Publishing.

Evans, K. \& Sullivan, J. M. (2000) Dual Diagnosis: Counseling the Mentally Ill Substance Abuser. New York: Guilford.

Fischer, G., Johnson, R. E., Eder, H., et al (2000) Treatment of opioid-dependent pregnant women with buprenorphine. Addiction, 95, 239-244.

Frischer, M., Collins, J., Millson, D., et al (2004) Prevalence of comorbid psychiatric illness and substance abuse in primary care in England and Wales between 1993-1998. Journal of Epidemiology and Community Health, in press.

Geerlings, P., Ansoms, C. \& van den Brink, W. (1997) Acamprosate and prevention of relapse in alcoholics. Results of a randomised, placebo-controlled, double-blind study in out-patient alcoholics in the Netherlands, Belgium and Luxembourg. European Addiction Research, 3, 129137.

George, T. P. \& Vessicchio J. C. (2002) Treating tobacco addiction in schizophrenia: where do we go from here? Addiction, 97, 795-796.

Gfroerer, J., Penne, M., Pemberton, M., et al (2003) Substance abuse treatment need among older adults in 2020: the impact of the aging baby-boom cohort. Drug and Alcohol Dependence, 69, 127-135.

Granholm, E., Anthenelli, R., Monteiro, R., et al (2003) Brief integrated outpatient dual-diagnosis treatment reduces psychiatric hospitalizations. American Journal on Addictions, 12, 306-313

Hamilton, S. P., Klimchak, C. \& Nunes, E. V. (1998) Treatment of depressed methadone maintenance patients with nefazodone. American Journal on Addictions, 7, 309-312.

Hawton, K., Arensman, E., Townsend, E., et al (1998) Deliberate self-harm: systematic review of efficacy of psychosocial and pharmacological treatments in preventing repetition. BMJ, 317, 441-447.

Hertzman, M. (2000) Divalproex sodium used to treat concomitant substance abuse and mood disorders. Journal of Substance Abuse Treatment, 18, 371-372.

Hunt, G. E., Bergen, J., \& Bashir, M. (2002) Medication compliance and comorbid substance abuse in schizophrenia: impact on community survival 4 years after a relapse. Schizophrenia Research, 54, 253-264.

Kiefer, F., Jahn, H., Tarnaske, T., et al (2003) Comparing and combining naltrexone and acamprosate in relapse prevention of alcoholism. Archives of General Psychiatry, 60, 92-99.

Kranzler, H. R., Burleson, J. A., Korner, P., et al (1995) Placebo-controlled trial of fluoxetine as an adjunction to relapse prevention in alcoholics. American Journal of Psychiatry, 152, 391-397.

Levin, F. R., Evans, S. M., Coomaraswammy, S., et al (1998) Flupenthixol treatment for cocaine abusers with schizophrenia: a pilot study. American Journal of Drug and Alcohol Abuse, 24, 343-360.

Lingford-Hughes, A., Potokar, J. \& Nutt, D. (2002) Treating anxiety complicated by substance misuse. Advances in Psychiatric Treatment, 8, 107-116.

Lingford-Hughes, A., Welch, S. \& Nutt, D. J. (2004) Evidencebased guidelines for the pharmacological management of substance misuse, addiction and comorbidity. Recommendations from the British Association for Psychopharmacology. Journal of Psychopharmacology, 18, 293-335

Littrell, K. H., Petty, R. G., Hilligross, N. M., et al (2001) Olanzapine treatment for patients with schizophrenia and substance abuse. Journal of Substance Abuse Treatment, 21, 217-221.

Malec, T. S., Malec, E. A. \& Dongier, M. (1996) Efficacy of buspirone in alcohol dependence: a review. Alcoholism: Clinical and Experimental Research, 20, 853-858.

Mason, B. J., Kocsis, J. H., Ritvo, E. C., et al (1996) A doubleblind, placebo-controlled trial of desipramine for primary alcohol dependence stratified on the presence or absence of major depression. JAMA, 275, 761-767.

McGrath, P. J., Nunes, E. V., Stewart, J. W., et al (1996) Imipramine treatment of alcoholics with primary depression. Archives of General Psychiatry, 53, 232-240.

Murray, R. M. (1980) Why are the drug companies so disinterested in alcoholism? British Journal of Addiction, 75, 113-115

National Institute for Clinical Excellence (2002) Guidance on the Use of Nicotine Replacement Therapy (NRT) and Bupropion for Smoking Cessation. Technology Appraisal Guidance no. 39. London: NICE.

Nunes, E. V., Quitkin, F. M., Donovan, S. J., et al (1998) Imipramine treatment of opiate-dependent patients with depressive disorders. A placebo-controlled trial. Archives of General Psychiatry, 55, 153-160.

Petrakis, I., Carroll, K., Gordon, L., et al (1994) Fluoxetine treatment for dually diagnosed methadone maintained opioid addicts: a pilot study. Journal of Addictive Diseases, 13, 25-32

Regier, D. A., Farmer, M. E., Rae, D. S., et al (1990) Comorbidity of mental disorders with alcohol and other drug misuse: results from the Epidemiologic Catchment Area Study. JAMA, 264, 2511-2518.

Schmitz, J. M., Averill, P., Stotts, A., et al (2001) Fluoxetine treatment of cocaine-dependent patients with major depressive disorder. Drug and Alcohol Dependence, 63, 207214

Schuckit, M., Irwin, M. \& Smith, T. L. (1994) One-year incidence rate of major depression and other psychiatric disorders in 239 alcoholic men. Addiction, 89, 441-445.

Scottish Executive (2003) Mind the Gaps: Meeting the Needs of People with Co-occurring Substance Misuse and Mental Health Problems. Edinburgh: Scottish Executive.

*Slattery, J., Chick, J., Cochrane, M., et al (2003) Prevention of Relapse in Alcohol Dependence. Health Technology Assessment Report no. 3. Glasgow: Health Technology Board for Scotland.

Stockwell, T., Hodgson, R., Edwards, G., et al (1979) The development of a questionnaire to measure severity of alcohol dependence. British Journal of Addiction to Alcohol and Other Drugs, 74, 79-87.

Sullivan, J. T., Skykora, K. \& Schneiderman, J. (1989) Assessment of alcohol withdrawal: the revised Clinical 
Institute Withdrawal Assessment for Alcohol Scale (CIWA-Ar). British Journal of Addiction, 84, 1353-1357.

Tyrer, P. \& Weaver, T. (2004) Desperately seeking solutions: the search for appropriate treatment for comorbid substance misuse and psychosis (editorial). Psychiatric Bulletin, 28, 1-2.

Zimmet, S. V., Strous, R. D. \& Burgess, E. S. (2000) Effects of clozapine on substance use in patients with schizophrenia and schizoaffective disorder. Journal of Clinical Psychopharmacology, 20, 94-98.

*Key reviews.

\section{Multiple choice questions}

1 The following medications have been shown to be helpful in the treatment of alcohol use disorders and depression:

a buspirone

b dothiepin

c paroxetine

d mirtazapine

e nefazodone.

2 As regards dual diagnosis:

a there is a dearth of studies in the primary care setting

$\mathrm{b}$ the USA has published much less research than the UK

c these patients visit general practitioners less frequently than does the general population d Scotland and England have the same dual diagnosis policy guidance

e there is clear evidence about which models of service work best.

3 Clozapine has been shown to be useful in the treatment of schizophrenia complicated by the use of:

a nicotine

b alcohol

c cannabis

d cocaine

e heroin.

4 The following are commonly prescribed for the treatment of opiate dependence:

a methadone

b buprenorphine

c naltrexone

d lofexidine

e heroin.

5 The following medications are licensed for use in 16- to 18-year-olds:

a buprenorphine

b bupropion

c methadone

d disulfiram

e acamprosate.

\section{MCQ answers}

\begin{tabular}{|c|c|c|c|c|}
\hline 1 & 2 & 3 & 4 & 5 \\
\hline a $F$ & a $\mathrm{T}$ & a $\mathrm{T}$ & a $\mathrm{T}$ & $\mathrm{a}$ \\
\hline $\mathrm{b} F$ & b F & $\mathrm{b} \mathrm{T}$ & $\mathrm{b} T$ & $b$ \\
\hline $\mathrm{F}$ & C F & c $\mathrm{T}$ & c F & \\
\hline $\mathrm{F}$ & d F & d F & d F & \\
\hline F & e $F$ & e $F$ & e F & \\
\hline
\end{tabular}

